\title{
Cut-off analysis of coherent vortical structure identification in a three-dimensional external flow
}

\author{
Anthi Miliou ${ }^{1}$, Iraj Mortazavi ${ }^{2}$, Spencer Sherwin $^{1}$ \\ ${ }^{1}$ Department of Aeronautics, Imperial College London, South Kensington \\ London, SW7 2BY, UK \\ ${ }^{2}$ Mathématiques Appliquées de Bordeaux UMR 5466 CNRS, Université Bordeaux 1 \\ 351 cours de la Libération, F-33405 Talence, France
}

\begin{abstract}
Vortical structure identification has more recently been applied in the study of transport of vortical structures in Low Reynolds Number Three-Dimensional Complex Geometries Flow. An important issue of this identification procedure is to choose an appropriate cutoff value which takes into consideration the finite precision of the flow results. This cutoff choice is studied in this work and applied to an external flow around a curved cylinder. The vortex identification technique at different cutoff values is compared to the more classical vortex threshold method showing the efficiency of choosing the optimal tolerance gap. The computations are done using a fully three-dimensional spectral $/ h p$ element method.
\end{abstract}

\section{Analyse des seuils de coupure pour identifier des structures cohérentes dans un écoulement tridimensionnel externe}

Resumé- Ce travail est consacré à l'identification des structures cohérentes présentes dans l'écoulement tridimensionnel d'un fluide visqueux aux bas nombres de Reynolds avec des géométries complexes. Une des issues importantes de ce processus d'identification est le besoin de spécifier un seuil numérique $\lambda_{2}$ pour tenir en compte des limites de précision de ce genre de calcul. Dans ce tarvail, ce seuil a été étudié et appliqué à un écoulement externe autour de tuyaux courbés. Les choix sont ensuite comparés aux résultats de la méthode plus classique du seuil de la vorticité mettant en évidence l'importance du choix approprié d'un seuil optimal. Tous les calculs sont effectués par une méthode tridimensionnelle du type "spectral/ $h p$ element".

Version abrégée francaise- L'identification des structures cohérentes représente un élément important dans la dynamique des écoulements réels. En effet, ces structures sont principalement convectées par l'écoulement, et constituent des entités qui demeurent quasi intactes au cours du temps. Elles transportent de plus une bonne partie de l'enstrophie [1]. Plusieurs stratégies d'identification existent. La plus naturelle consiste en la visualisation des isovaleurs de la vorticité. Si cette première méthode permet de se faire une bonne idée de la localisation des tourbillons, elle n'est toutefois pas suffisamment précise dès qu'il s'agit d'isoler ces tourbillons. Un critère de détection utilisant un seuil de vorticité fixé aussi, à priori, ne donne pas des résultats fiables. En effet, si par définition, il permet d'isoler les parties de l'écoulement dans lesquelles la vorticité est la plus forte (en valeur absolue), il n'arrive pas toujours à distinguer les zones cohérentes des zones non cohérentes. Les figures $2(\mathrm{e})$ et $2(\mathrm{f})$ montrent comment ce type de détection confond des zones cisaillées non-cohérentes avec des structures concentrées, transportées dans l'écoulement. Hussain a alors proposé [4] une méthode plus satisfaisante pour un fluide incompressible, qui consiste à identifier les noyaux tourbillonnaires en fonction du principe de pression minimale dans la rotation d'un fluide parfait. Cela revient à considérer la nature des valeurs propres du tenseur des gradients de vitesse : la deuxiéme plus grande valeur propre de $\boldsymbol{S}^{2}+\boldsymbol{\Omega}^{2}$ devrait être négative, pour qu'on se situe dans le noyau d'un tourbillon. $\boldsymbol{S}$ et $\boldsymbol{\Omega}$ correspondent aux composants symmétrique et antisymmétrique du tenseur de gradient de la vitesse. Comparé à l'usage direct des isocontours de vorticité, ce critère a l'avantage d'isoler des 
structures rotationnelles telles que des tourbillons, de zones non-cohèrentes (la couche limite, ...) et irrotationnelles. Pour effectuer cette ètude, on utilise une simulation numérique directe de l'écoulement d'un fluide visqueux incompressible autour d'une conduite cylindrique courbée (figure 1) par une méthode tridimensionnelle du type "spectral/hp element" [5].

On montre que, même si le critère de Hussain ne nécessite aucun seuil théorique donné a priori, il est cependant nécessaire de spécifier un seuil numérique pour sa mise en oeuvre. La détermination de ce paramètre est effectuée en considérant le pourcentage d'enstrophie contenue dans les zones cohérentes détectées par le critère de Hussain en fonction du paramètre $\lambda_{2}$ (figure 3). Trois différents comportements sont alors observés : pour des très petites valeurs du seuil, proche de la définition initiale, les résultats sont dominés par le bruit numérique qui rend le critère inutilisable (figure 2(a) et figure 3). Ensuite, l'apparition d'une zone intermédiaire optimale identifiant des structures cohérentes présentes dans l'écoulement est constatée (figure $3)$. Dans cette gamme, plus on accroit la valeur de $\lambda_{2}$, plus la taille des zones rotationnelles identifiées se voit réduite (figure 2(b) et figure 2(c)). Finalement, une région asymptotique est constatée. Cette région correspond physiquement aux couches de cisaillement contenant des très grandes quantités de l'enstrophie sans pour autant correspondre à une structure cohérente (figure 2(d) et figure 3). Ces résultats confirment l'existence d'une région optimale intermédiaire pour le choix de $\lambda_{2}$ en dehors de laquelle on sera confronté soit aux bruits confondants des structures cohérentes et non-cohérentes, soit à un manque d'informations sur la vorticité. Ce critère de détection, ayant finalement été validé, pourrait être utilisé de façon pertinente afin d'établir certaines propriétés intrinsèques aux tourbillons, en fonction du nombre de Reynolds : nombre et taille des tourbillons, pourcentage d'enstrophie de l'écoulement qu'ils transportent, etc.

\section{Introduction}

Bluff bodies are defined as those for which the flow separates from a large section of the body's surface [2]. Bluff bodies are encountered in many engineering applications i.e. offshore riser pipes, bridges, heat exchanger tubes etc. The study of the flow past these bodies is of high importance both in the research community and in the industrial world.

Once the boundary layers separate, they then become free shear layers. Above a bodydependent critical Reynolds number, the shear layers interact and roll up resulting in vortices being shed from alternating sides of the bluff body. This produces the formation of two rows of discrete vortices in a staggered array in the wake commonly known as von-Kármán vortex street and this process is called vortex shedding.

The shedding of vortices can cause large unsteady forces of high amplitude which under some circumstances can yield a structural failure. As a result, the study of vortex shedding and transport has received significant attention from the research communities over the past century.

One of the most helpful techniques to study two and three-dimensional vortex dynamics is the identification of the Coherent structures (CS) [3], [4]. Coherent structures generally refer to the organized and concentrated rotational patterns within the flow and are a useful way to characterize flow evolution in time. An important feature of coherent structures identification is the reduction of a vector flow field into scalar quantity. Typically a specific isocontour value, or cuttoff, of the scalar quantity is then chosen to identify the coherent structures. Whilst the original definitions [3], [4] defined exact values for the cutoff in numerical practice the cuttoff value is varied to eliminate numerical noise.

In this work, we focus on the choice of cutoff and its effect on the identification procedure extending previous research [9] to a three-dimensional flow: the external flow past a curved circular 
cylinder which generates a rich vortex shedding pattern. The identification procedure for several cutoffs is studied and compared to the more classical vortex threshold method. The asymptotical behaviour of the enstrophy values versus the cutoff choice is then studied to determine the most optimal cutoff ranges. All computations are performed employing a $3 \mathrm{D}$ spectral $/ h p$ element method.

\section{Numerical method}

The mesh generation for all the computational investigations was accomplished with Felisa where a modified advancing layers method is employed near the pipe wall regions and a method based on the advancing front technique is employed for the rest of the domain [8].

Computations have been performed using Nektar [5], [6] a three-dimensional incompressible Navier-Stokes solver, based on the spectral/hp element method. Spectral/ $h p$ methods are highorder discretisation methods where one can increase simultaneously the number of elements (h-refinement) in the domain and the order, $\mathrm{P}$, of the polynomial expansions in each element (p-refinement). In the current work this discretisation has been used with unstructured and hybrid shape conforming subdomains.

To discretise the incompressible Navier-Stokes equations a high-order stiffly stable splittingscheme [7] is employed where the non-linear terms are solved explicitly and the linear terms are solved implicitly. This high-order splitting-scheme can be interpreted as propagating the velocity and pressure fields over a time step $\Delta t$ in three substeps. The first step explicitly advances the non-linear advection terms, subsequently a Poisson equation for pressure is solved which imposes the divergence-free condition on the intermediate velocity field from the first step. Finally Helmholtz's systems are solved for each velocity component to impose viscous linear terms. Therefore the full scheme requires the solution of a Helmholtz equation for each component of the velocity fields and a Poisson equation for the pressure field. We note that all of the results presented were produced by the three-dimensional code where the spectral $/ h p$ element discretisation was adopted in all three Cartesian directions.

All the flow fields obtained with Nektar are solutions to the Navier-Stokes equations using higher order polynomial expansions. Linear finite elements are however employed for the postprocessing utilities. The integral of $\omega^{2}$ in the domain was calculated with linear shape functions $N_{i}$ in three-dimensions by first decomposing each element to a large number of smaller tetrahedra. If the original element is a tetrahedron for example, then it can be decomposed to $n^{3}$ similar smaller tetrahedra with $n+1$ equispaced interpolating points along each edge of the original element. A better resolution of the non-smooth function in space is enabled this way.

\section{$3 \quad$ Identification and cut-off analysis}

In order to investigate the wake structures developed by the external flow past a curved cylinder, the $\lambda_{2}$ criterion presented by Jeong and Hussain [4] was used for identifying the vortex cores in the wake. The $\lambda_{2}$ criterion stems from the pressure minimum criterion due to inviscid flow rotation and dictates that the second largest eigenvalue of $\boldsymbol{S}^{2}+\boldsymbol{\Omega}^{2}$ must be negative in order to locate a vortex core. $\boldsymbol{S}$ and $\boldsymbol{\Omega}$ are the symmetric and antisymmetric components of the velocity gradient tensor, i.e.

$$
\mathbf{S}_{i, j}=\frac{1}{2}\left(\frac{\partial u_{i}}{\partial x_{j}}+\frac{\partial u_{j}}{\partial x_{i}}\right), \quad \boldsymbol{\Omega}_{i, j}=\frac{1}{2}\left(\frac{\partial u_{i}}{\partial x_{j}}-\frac{\partial u_{j}}{\partial x_{i}}\right),
$$


This identification is more insightful than the direct use of vorticity isocontours because it isolates rotational structures, such as vortices, from boundary layer and other non-rotational shear features.

To non-dimensionalise $\lambda_{2}$ we define $\lambda_{2}^{\star}=\lambda_{2} D / U$ where $D$ and $U$ are a characteristic length and velocity of the problem which for this case are taken as the cylinder diameter and free stream velocity. Although the formal definition of the vortex identification is when $\lambda_{2}^{\star}=0$, numerically it is necessary to determine a "tolerance" for the cutoff value. The determination of an appropriate cut-off value can be performed numerically and has previously been investigated in two-dimensional flows [9] [10]. In this work Creusé and Mortazavi identified an optimal cutoff value for small perturbations in a laminar flow over a dihedral plane. They showed that the optimal cutoff should be large enough to remove the numerical noise of the background flow, and small enough to distinguish the ensemble of vortical structures.

Hussain notes that since $\boldsymbol{S}^{2}+\boldsymbol{\Omega}^{2}$ is symmetric, it has real eigenvalues only. Then, he defines a vortex core when $\lambda_{2}$ is negative as we say in the previous paragraph. So, should be careful saying "Although the formal definition of the vortex identification is when $\lambda_{2}=0$ ". $\lambda_{2}=0$ demarcates the boundary between no vortex cores and all vortex cores together.

In figure 2 we show the $\lambda_{2}^{\star}$ coherent structures generated by the three-dimensional flow past a curved cylinder. This geometry is effictively a quarter ring of 12.5 non-dimensional radius of curvatur e that has an artificial blending applied to it towards the bottom and ends semisubmerg ed into the ground. The flow is into the page. Figure 1 shows this geometry. For this case, a shear velocity profile and a viscous wall were applied. Specifically, as we view Figure 1, the top boundary and the left boundary would be a symmetry boundary condition. The bottom boundary would be a viscous wall $\left[\mathrm{V}=\begin{array}{lll}0 & 0 & 0\end{array}\right]$. The right boundary would be a free-stream boundary condition. The inflow and outflow planes are parallel to this page. An exponentially decaying shear ed profile was chosen for the planes having a free-stream boundary condition. The sheared profile is: $v=1-e^{-(z+12.5)}$.

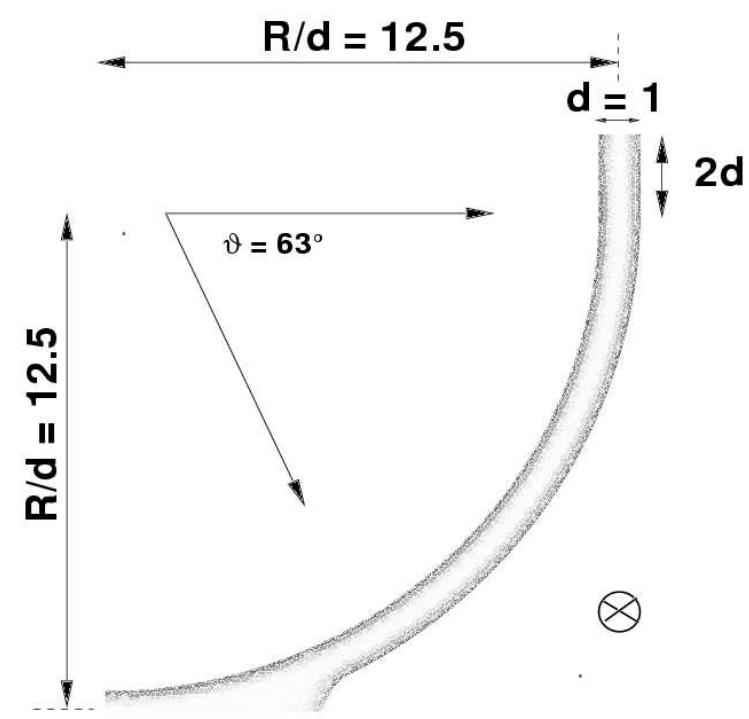

Figure 1: Definition of body, $R / d=12.5$. Artificial blend applied on sections of the body that correspond to angles larger than $63^{\circ}$ from the horizontal.

Different compared cut off values are (a) $\lambda_{2}^{\star}=-0.001$, (b) $\lambda_{2}^{\star}=-0.1$, (c) $\lambda_{2}^{\star}=-0.3$, (d) $\lambda_{2}^{\star}=-1$. We note that at very low values close to the analytical definition, as shown in figure 2(a), the structures are dominated by numerical noise. However within the range - 
$0.1 \leq \lambda_{2}^{\star} \lambda-0.6$ we observe a more optimal representation of the coherent structure capturing the three-dimensional vortex shedding from the curved cylinder without being obscured by the numerical noise. However for even higher values of $\lambda_{2}^{\star}$, as shown in figure 2(d), we observe that only the most energetic shear layers are captured by the $\lambda_{2}^{\star}$ isocontour. Furthermore, 2(e) and (f) depict normalised enstrophy isocontours at values of 0.363 (average) and 1.2. The difference between the vortex core identification criterion, that captures only vortical structures, and the magnitude of vorticity is thus highlighted.

To try and quantify the features observed in figure 2 we have calculated the proportion of the enstrophy captured within the coherent stuctures using the $\lambda_{2}^{\star}$ definition. This has then been normalised by the total enstrophy within the global flow domain and plotted as a function of the cut-off value as shown in figure 3. In this figure we show the same data plotted respectively on Log-Log and Lin-Log axis. They confirm basically the previous comments revealing other potential cutoffs. As the figure suggests, three different behaviours are distinguishable. For $\left|\lambda_{2}^{\star}\right|<0.1$ there is a small region of high negative slope where a very large percentage of the total enstrophy is captured. This region corresponds to the numerically noisy vorticity fields as shown in figure (2a). Next we observe change in slope of the log-log curve 3(b) between $0.1<\left|\lambda_{2}^{\star}\right|<0.6$ where the captured enstrophy corresponds to figures 2(b) and 2(c). In this range the captured enstrophy decreases with the cut-off magnitude. As the cut-off is increased only the more energetic near wake structures are resolved, as can be seen in figures 2(b) and (c), thereby eliminating large regions of relatively low enstrosphy in the far wake structures. Finally, for $\left|\lambda_{2}^{\star}\right|>0.6$, a third region is evident with a different slope that corresponds to the very energetic shear layers near the cylinder body as shown in figure 2(d). This implies that increasing $\lambda_{2}^{\star}$ above an optimal range of cut-off values generates an abrupt loss of information affecting all structures outside of the shear layers which contain the highest enstrophy.

\section{Conclusions}

In this work a three-dimesional spectral $/ h p$ element method was used to simulate an external flow around a three-dimensional curved cylinder. This 3D low Reynolds number flow generates complex vortical structures and to investigate the wake structures the $\lambda_{2}^{\star}$ criterion of Jeong and Hussain [4] has been applied. As noted by Jeong \& Hussain, this technique is preferred to vorticity magnitude which is unable to distinguish between boundary layers and vortical structures.

The main part of this work was focused on the choice of a cut-off range of the $\lambda_{2}$ coherent vortical structure identification. As a procedure for choosing an appropriate cut-off the proportion of the enstrophy captured within the $\lambda_{2}$ coherent structure to the total enstrophy within the global flow domain was studied. Three different behaviours were distinguished relating the coherent structure to the cutoff value. At very low values of the cut-off, close to the idealised definition, the results are dominated by numerical noise thereby making the criterion inapplicable.

An optimal intermediate region was observed which captured a decreasing amount of the wake structures as the cut-off value was increased. Finally an asymptotic region was observed where the captured enstrophy varied relatively slowly with the cut-off value. This region physically corresponds to capturing only the shear layers containing the highest enstrophy of the flow.

In conclusion we propose that numerical application of the $\lambda_{2}$ coherent structure identification criterion should be used in conjunction with an enstrophy capture analysis to determine a valid range of non-zero cut-off values. 


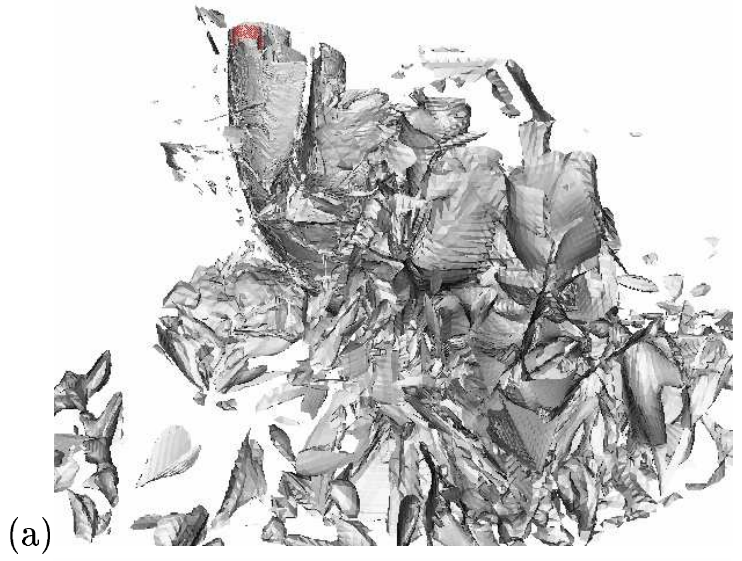

(b)
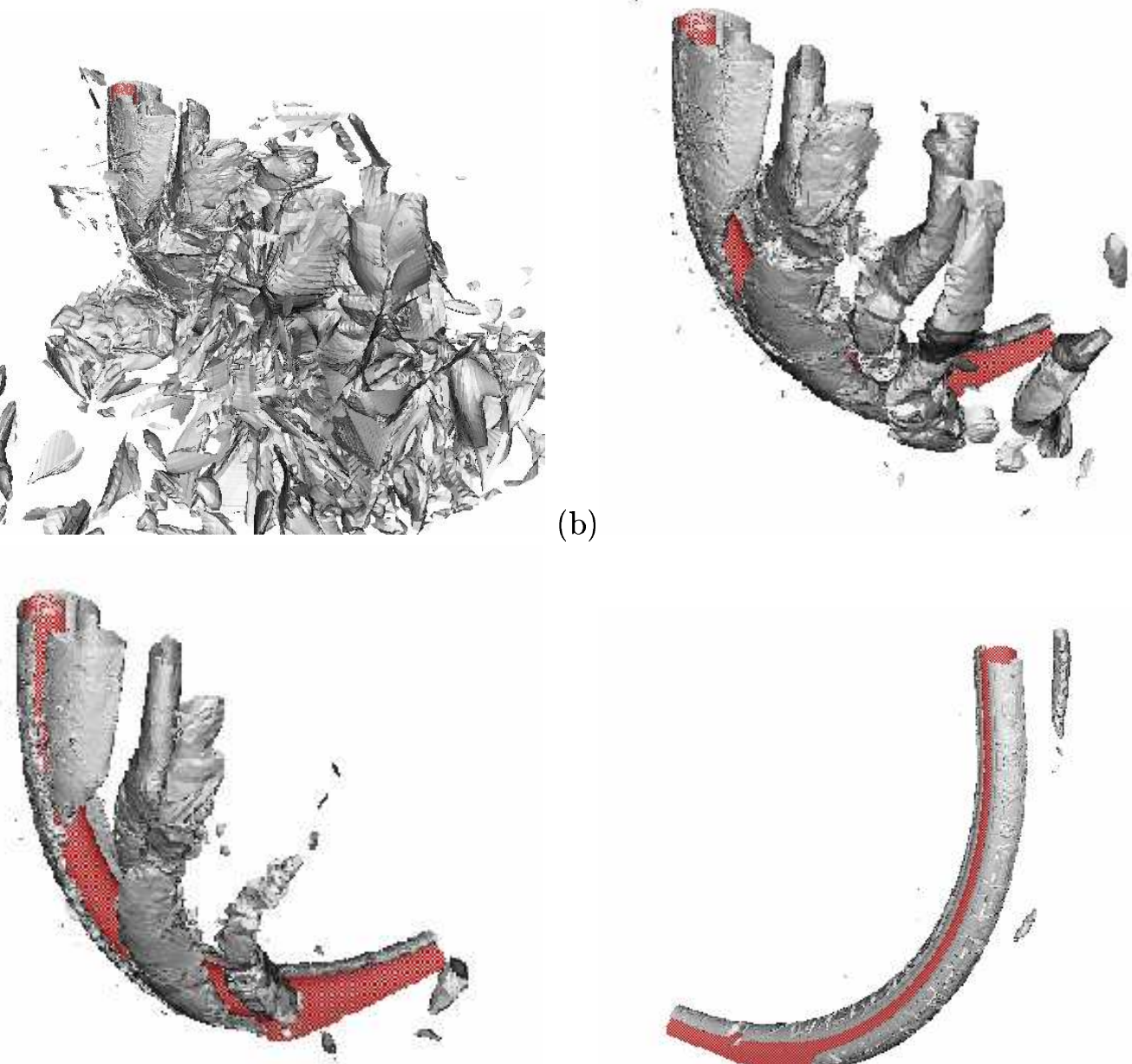

(d)

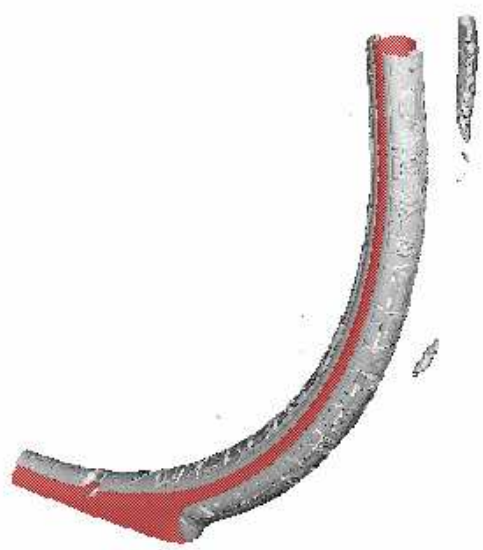

(c)

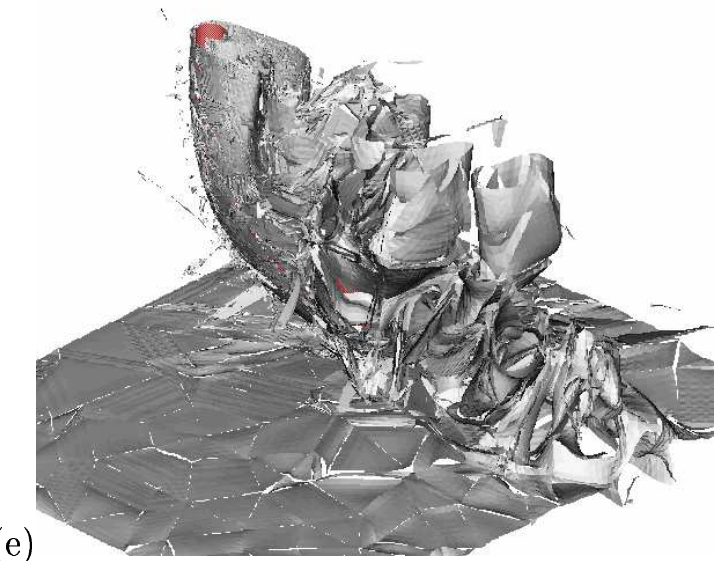

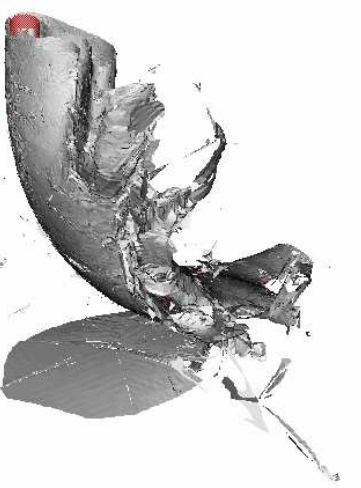

(f)

Figure 2: $\lambda_{2}^{\star}$ isocontour at (a) $\lambda_{2}^{\star}=-0.001$, (b) $\lambda_{2}^{\star}=-0.1$, (c) $\lambda_{2}^{\star}=-0.3$, (d) -1 . Normalised enstrophy isocontour at a value of (e) 0.363 and (f) 1.2 
(a)

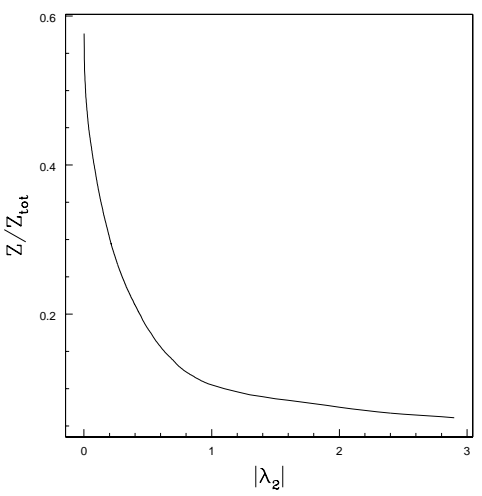

(b)

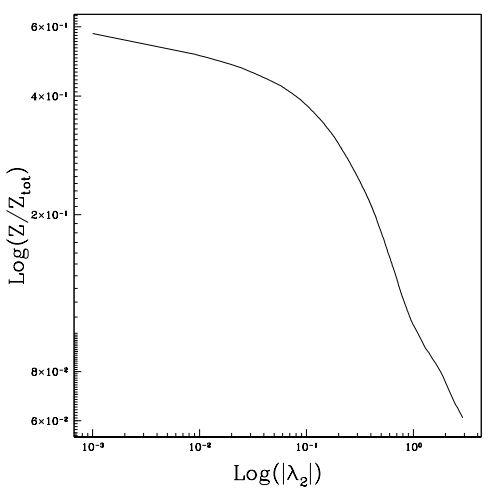

(c)

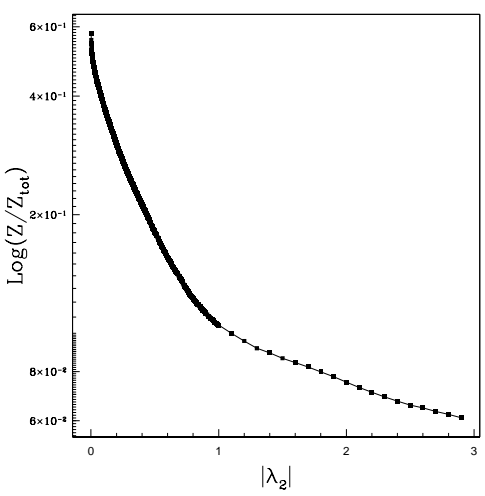

Figure 3: (a) Normalised enstrophy versus $\lambda_{2}$, (b) Log-Log axis (b) lin-Log axis

\section{References}

[1] C. Basdevant and T. Philipovitch, 'On the "Weiss criterion" in two-dimensional turbulence', Physica $D, 73,17-30$ (1994).

[2] R.D. Blevins, 'Flow-induced vibration', Van Nostrand Reinhold Company, (1977).

[3] J. Weiss, 'The dynamics of enstrophy transfer in two-dimensional hydrodynamics', Physica D, 48, 273-294 (1991).

[4] J. Jeong and F. Hussain, 'On the identification of a vortex', J. Fluid Mechanics, 285, 69-94, (1995).

[5] S.J. Sherwin \& G.E. Karniadakis, 'Tetrahedral $h p$ finite elements: Algorithms and flow solutions', Journal of Computational Physics, 124, 14-45 (1996).

[6] G.E. Karniadakis \& S.J. Sherwin, `Spectral/hp Element Methods for CFD`, Oxford University Press (1999).

[7] G.E. Karniadakis, M. Israeli, \& S.A. Orszag, 'High-order splitting methods for the incompressible Navier-Stokes equations`, Journal of Computational Physics, 97, 414-443 (1991).

[8] J. Peraire, J. Peiro \& K. Morgan, 'Multigrid solution of the 3-D compressible Euler equations on unstructured tetrahedral grids', Int. J. Numer. Methods Eng., 36, 1029-1044 (1993).

[9] E. Creusé and I. Mortazavi 'Vortex dynamics over a dihedral plane in a transitional slightly compressible flow: a computational study', Europ. J. Mech. B/Fluids, 20. 603-626 (2001).

[10] E. Creusé and I. Mortazavi 'Identification of concentrated structures in slightly compressible twodimensional flows' C. R. Ac. Sciences. Paris, 5 693-699 (2001). 